\title{
A Methodology for Studying Cognitive Groupings in a Target Tracking Task
}

\author{
Steven J. Landry, Thomas B. Sheridan, IEEE and Yan Yufik
}

\begin{abstract}
Subjects performing visual target tracking tasks have been shown to utilize perceptual organization. This organization has both Gestalt and goal-oriented features. Previous studies have attempted to use memory recall techniques to examine potential cognitive groupings in air traffic control (ATC), which is, in part, a complex target tracking task. In the present research, a special form of cluster analysis was successful in revealing cognitive groupings having appreciable influence on task performance in a targettracking task designed to resemble ATC. The method of cluster analysis was derived from the "virtual associative network" model of memory organization, and applied to the record of eye fixations in the course of task performance. Results using inexperienced subjects showed fixation clustering consistent with Gestalt factors. Task objectives (such as conflict detection) did not seem to affect grouping as much. The subjects' recall was generally poor, except where direct manipulation of targets occurred. We conclude that a) cognitive grouping influences performance, $b$ ) recall techniques may not be able to elicit subjects' cognitive groupings, and c) such groupings can be determined via analysis of eye fixations. These findings have implications for studying workload assessment and information structuring in complex visual scanning tasks.
\end{abstract}

Index terms - Air traffic control, cognitive grouping, eye fixations, memory organization.

\section{INTRODUCTION}

\section{A. Cognitive grouping}

Miller [1] demonstrated that the span of immediate memory for one-dimensional stimuli is $7 \pm 2$ items. Item grouping into "chunks" allows people to overcome this processing bottleneck. Numerous experiments have demonstrated cognitive grouping, from relatively simple list-recall tasks [2], to complex schemes employed by chess players. Gestalt psychologists identified proximity, similarity, common motion, symmetry and good form ("Prägnanz") as the main factors underlying feature grouping in visual perception. In addition, changes in the task influenced grouping, particularly when accompanied by reward/punishment alterations [3].

\section{B. Target Tracking}

Pylyshyn and Storm [4] demonstrated that individual targets in a simple target-tracking task were being cognitively "indexed" based on Gestalt factors, allowing the tracking of targets independently of the visual task. Yantis [5] proposed that the targets are attended, but that they are grouped into unified "virtual objects," and it is these virtual objects that are tracked. Yantis duplicated the Pylyshyn and Storm experiments, but varied the Gestalt factors that influence formation and maintenance of the virtual clusters. His results validated the hypothesis that perceptual grouping can be influenced by both the Gestalt factors and by the subject's objectives.

Since air traffic control (ATC) is at least partially a target-tracking task, one would expect the same influences to manifest in control performance. Means et al. [6] found evidence that aircraft are grouped in memory with related aircraft. Dougherty et al. [7] suggested that some space-based attentional schema underlie grouping, and attempted to elicit these groupings by testing recall. However, these tests failed to identify any groupings, and the conclusion was drawn that there exist no aircraft-toaircraft links.

\section{Detecting clusters by recall}

One difficulty in using recall information in ATC tasks is the inability of even skilled subjects to recall some types of information. Information such as aircraft type, call sign, and groundspeed, is recalled less than $40 \%$ of the time (see [6] and [8]). Information that is of more direct value to the controller's task, however, such as the position of aircraft, the initial alphabetic part of a call sign, and control level (whether the aircraft was in the sector, about to enter, etc.) was recalled more successfully, but $10 \%$ to $30 \%$ were still missed.

Additionally, since the normal workload level experienced by controllers is rather high, it seems unlikely that extensive memorization is occurring. Yet the earlier target tracking work, and the fact that the controllers do their job as well as they do with the number of aircraft they are required to track suggests that some type of cognitive organization is occurring.

Copyright (C) 2001 IEEE. Reprinted from IEEE Transactions on Intelligent Transportation Systems Volume 2 Number 2. This material is posted here with permission of the IEEE. Internal or personal use of this material is permitted. However, permission to reprint/republish this material for advertising or promotional purposes or for creating new collective works for resale or redistribution must be obtained from the IEEE by sending an email message to pubs-permissions@ieee.org. 


\section{Detecting clusters by eye movements}

An alternate method of determining clusters is to examine eye movements. Most visual information is received from the high-resolution, small field-ofview foveal region in the retina. Given the vision system's transmission limitation (one estimate is $40 \mathrm{~K}$ bits per second [9]), a sequence of fixations and saccades must occur for a person to perceive a complex scene. A realistically complex scene contains items or areas of relatively high interest or content, and areas of little interest or content. Most items must be fixated on for a period of time to process the level of detail required. Normally, eye fixations keep switching from one feature to another until the entire scene is viewed.

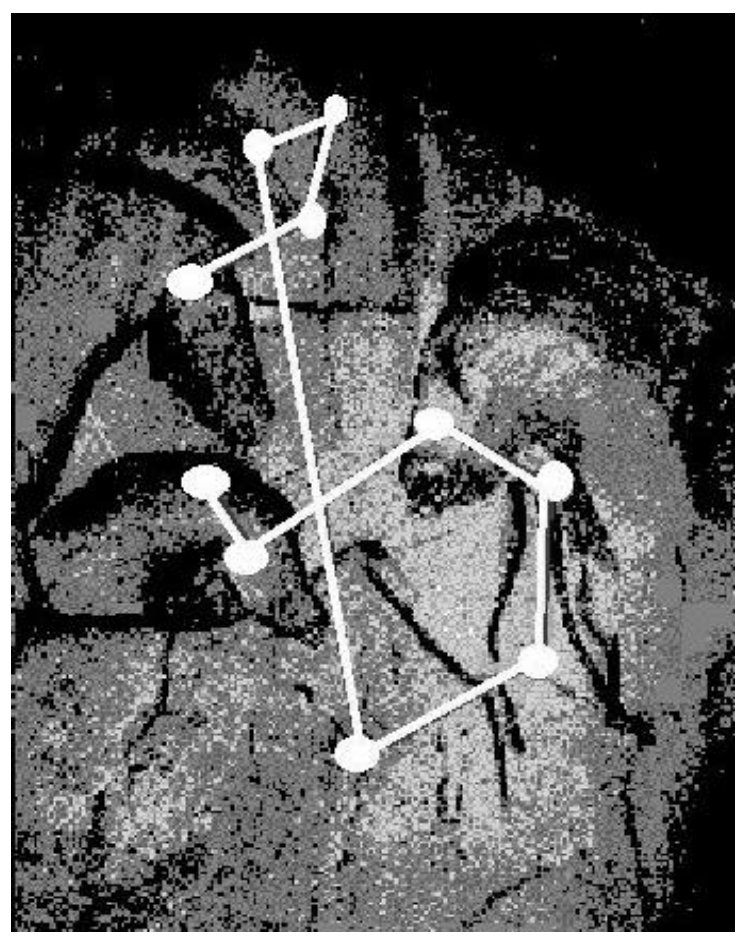

Figure 1. Scanpath [11]

Noton and Stark [10] showed that sequences of fixations were "idiosyncratic" and "repetitive", and referred to this sequence as a "scanpath". Subjects' eye movements demonstrated repeated fixations on particular items of interest in the scene, and the pattern for revisiting those items was different for different subjects, while remaining relatively stable for the same subject. An example of a scanpath is seen in Figure 1 [11]. The white ovals are fixations; the white lines are saccades. If the same subject viewed this or a similar picture again, the scanpath would be similar. For a different subject, however, the scanpath could be significantly different.
One theory is that features of the stimulus control fixations. Our eye is drawn to areas of contrast or some other features distinct from the object as a whole. By contrast, the scanpath theory holds that viewing is directed by an internal model of the object updated in the course of fixations. Convincing support for the theory comes from experiments in [12]. Subjects viewed three different patterns of Xs, then were first asked to visualize the patterns on a blank screen, then draw the patterns on a blank screen. Similarity analysis of the visualized object's scanpath showed strong agreement with the scanpath of the viewed patterns. No stimulus was available to the eye or brain when visualizing, yet nearly the same scanpath resulted, confirming that a top-down cognitive model was the source.

\section{E. Virtual Associative Network}

Our experiments were motivated by the "Virtual Associative Network" (VAN) model [13] - [15] proposed as a means to understand cognitive mechanisms responsible for the performance of the subjects in detecting potential conflicts. According to the VAN model, the subject's internal representation (internal model) of the air traffic situation develops in the course of multiple fixations and involves two processes: integration of all the perceived aircraft relationships into a unified network encompassing the entire visual scene ("associative network"), and dynamic network partitioning into internally cohesive and externally weakly coupled "clusters." The concept of clusters is resonant with that of "virtual objects" in Yantis suggesting that aircraft in a cluster are perceived as a cohesive relational unit distinct from the other units in the scene. As the scene changes (e.g. aircraft change trajectories and/or enter and leave the sector), the network is re-organized and the clusters change accordingly (hence, "virtual clusters."). The model is discussed in more specific detail below as relates to visual scanning of aircraft on an ATC display.

The model is accompanied by software that rapidly detects the formation of clusters. Although in this research the analysis of clusters was performed after the data was collected, the software has the capability to perform this analysis in real-time. This capability may allow for future experiments to change features that may influence the formation or maintenance of clusters.

\section{TARGET TRACKING EXPERIMENT}

\section{A. Method}

Given the evidence that top-down perceptual organization is possibly occurring in target tracking 
Table 1. Experiment Matrix

\begin{tabular}{c|lcc}
\hline Scenario number & \multicolumn{1}{c}{ Configuration } & Co-altitude crossings & Actual conflicts \\
\hline 1 & Sequenced & 25 & 5 \\
2 & Random & 18 & 3 \\
3 & Sequenced & 10 & 0 \\
4 & Canonical & 17 & 1 \\
5 & Random & 15 & 0 \\
6 & Random & 18 & 7 \\
7 & Canonical & 16 & 5 \\
8 & Sequenced & 15 & 2 \\
9 & Canonical & 11 & 1 \\
\hline
\end{tabular}

tasks, and that eye movements may be indicative of this organization, an experiment was performed at the Human-Machine Systems Lab at M.I.T. to attempt to identify any relationship cluster members may have with some objective criteria, such as recall, performance, or task objectives. The task was to identify targets predicted to conflict (defined as targets at the same "altitude" that pass within a particular distance on the display of one another).

Eighteen subjects were recruited to participate in the experiment. Subjects were undergraduate or graduate students at M.I.T. Four subjects could not be calibrated and were dismissed from the experiment. The remaining fourteen subjects were generally untrained in air traffic control, although one subject had 140 flying hours, one subject had 70 flying hours, and those two subjects as well as three others reported some experience with air traffic control concepts. Subjects were paid $\$ 15.00$ for their participation. No incentives for performance were used. Before beginning the experiment, subjects read a written set of instructions that also included a picture of the display with which they would be interacting. Next, a verbal description of the experiment was given, with a detailed explanation of the display, the controls, and the responsibilities of the subject. Subjects were then asked if they had any questions. Finally, subjects performed a five-minute sample trial to familiarize them with the task. A few subjects had some remaining confusion with the keyboard controls at the end of this trial and an additional sample trial was performed.

Subjects were told that the task was similar to air traffic control in that they were to identify conflicting targets. They were instructed to identify conflicts as soon as they believed the targets would conflict. A conflict was any time two targets at the same altitude passed within 5 NM of each other. A ring the subjects could have displayed around a target identified this range. A conflict was identified by clicking on the targets and selecting a menu item.

The subjects were also instructed that they were responsible for two "secondary" tasks. The first of these tasks was to "accept" targets as they appeared on the screen, accomplished by using a mouse to move a cursor to and click on the target. The second task was to hand off the targets just before they left the screen, accomplished by selecting the target and a menu item. The subjects were instructed, however, that their primary task was to identify conflicts.

Finally, the subjects were told that there would be two recall questionnaires that would appear at the end of the scenario, but that they need not attempt to recall any particular information.

Nine scenarios, shown in Table 1, were developed. The number of targets (aircraft) remained constant (at 14) to remove this effect from performance. Since the formation of clusters is often associated with Prägnanz ("good form"), three of the scenarios contained canonical configurations of traffic. These canonical shapes were aircraft appearing to fly in diamond-shaped or triangular formations. In three other scenarios, many targets were sequenced, appearing to fly along predefined routes with other targets. The remaining three scenarios had targets' positions and directions of flight randomly selected.

The number of conflicts was varied between high ( 5 or 7 ), low ( 1 to 3 ) and none. Targets were randomly assigned a constant altitude at flight level (FL) 310 (31,000 feet), FL330, FL350, FL370 or FL390. Ground speeds ranged from 340 to 480 knots.

All acceptances, handoffs, and detections (predictions) of conflicts were recorded, including the scenario time for each action. An Iscan, Inc. Eye Movement Monitoring System was used to record eye movement data. This system consists of a standard video camera attached to a pan and tilt unit 
Table 2. General Questions

\begin{tabular}{|c|l|}
\hline Number & \multicolumn{1}{|c|}{ Question } \\
\hline 1 & How many conflict pairs were there? \\
\hline 2 & How many targets did you accept? \\
\hline 3 & How many targets did you hand off? \\
\hline 4 & What was the predominant direction of flight? \\
\hline 5 & What was the most common altitude? \\
\hline 6 & In what direction were the targets moving the fastest? \\
\hline 7 & In what portion of the screen were there conflicts? \\
\hline 8 & How many targets were traveling south, including southeast and southwest? \\
\hline 9 & How many targets were traveling north, including northeast and northwest? \\
\hline 10 & How many targets were traveling east, including southeast and northeast? \\
\hline 11 & How many targets were traveling west, including northwest and southwest? \\
\hline 12 & How many targets were at FL390? \\
\hline 13 & How many targets were at FL310? \\
\hline 14 & How many targets were traveling above 440 knots? \\
\hline 15 & How many targets were traveling below 360 knots? \\
\hline
\end{tabular}

which images the pupil of the subject's eye, an infrared illuminator and two monitors for viewing the subject's eye and subject's scene.

The VAN-based software computed aircraft clusters, as follows. In the association network, nodes were formed as a result of eye fixation on the aircraft and links as a result of eye transition between the aircraft. Links were being weighted based on the cumulative fixation duration on the corresponding nodes (each fixation-transition-fixation sequence causes weight increment). Clusters are formed by network partitioning so that the sum of the link weights internal to every group of nodes exceeds the weights of all links from that group to external nodes. Essentially the method returns clusters of aircraft subjected to joint viewing via repetitive patterns of fixations and transitions internal to the clusters. According to the model, partitioning complex situations into a small number of such clusters facilitates comprehension.

Once the trial time limit was reached, target details were erased and two questionnaires appeared. The first questionnaire asked specific questions about three targets selected ahead of time by the experimenter. The questions were specific to the target, and asked about speed, altitude and with which other targets the subject target conflicted. The second questionnaire contained three questions, which were selected pseudo-randomly from a list of 15 questions. The list of questions is shown in table 2. The questions were designed to be general questions about the scenario, rather than specific to any one target.

\section{B. Results}

Conflict detection performance was poor in the task. A preliminary experiment had demonstrated that 12 targets were difficult for untrained subjects to track, and 18 were nearly impossible. In the current experiment subjects appeared to have difficulty at 14 targets per scenario. In comparison, air traffic controllers generally deal with less than 14 targets at a time. The scenario was made deliberately difficult so that performance changes could be more easily detected in a reasonable number of trials. The number of correct detections (CD) and false alarms (FA) were low, as shown in Table 3.

Table 3. Correct Detections and False Alarms

\begin{tabular}{|c|c|c|}
\hline Run ID & CD Rate & FA Rate \\
\hline Scenario 5 & NA & $0.94 \%$ \\
Scenario 3 & NA & $4.11 \%$ \\
Scenario 4 & $78.6 \%$ & $1.32 \%$ \\
Scenario 2 & $57.1 \%$ & $1.87 \%$ \\
Scenario 8 & $50.0 \%$ & $2.15 \%$ \\
Scenario 7 & $32.9 \%$ & $1.91 \%$ \\
Scenario 6 & $26.5 \%$ & $3.75 \%$ \\
Scenario 1 & $15.7 \%$ & $3.45 \%$ \\
Scenario 9 & $14.3 \%$ & $2.78 \%$ \\
\hline
\end{tabular}

When the probabilities of correct detection (CD) and false alarm (FA) are cross-plotted, the curve in Figure 2 is generated. This figure is similar to a receiver-operating characteristic (ROC) curve, used in signal detection theory. The ROC curve has been used to analyze the performance of alerting systems, 


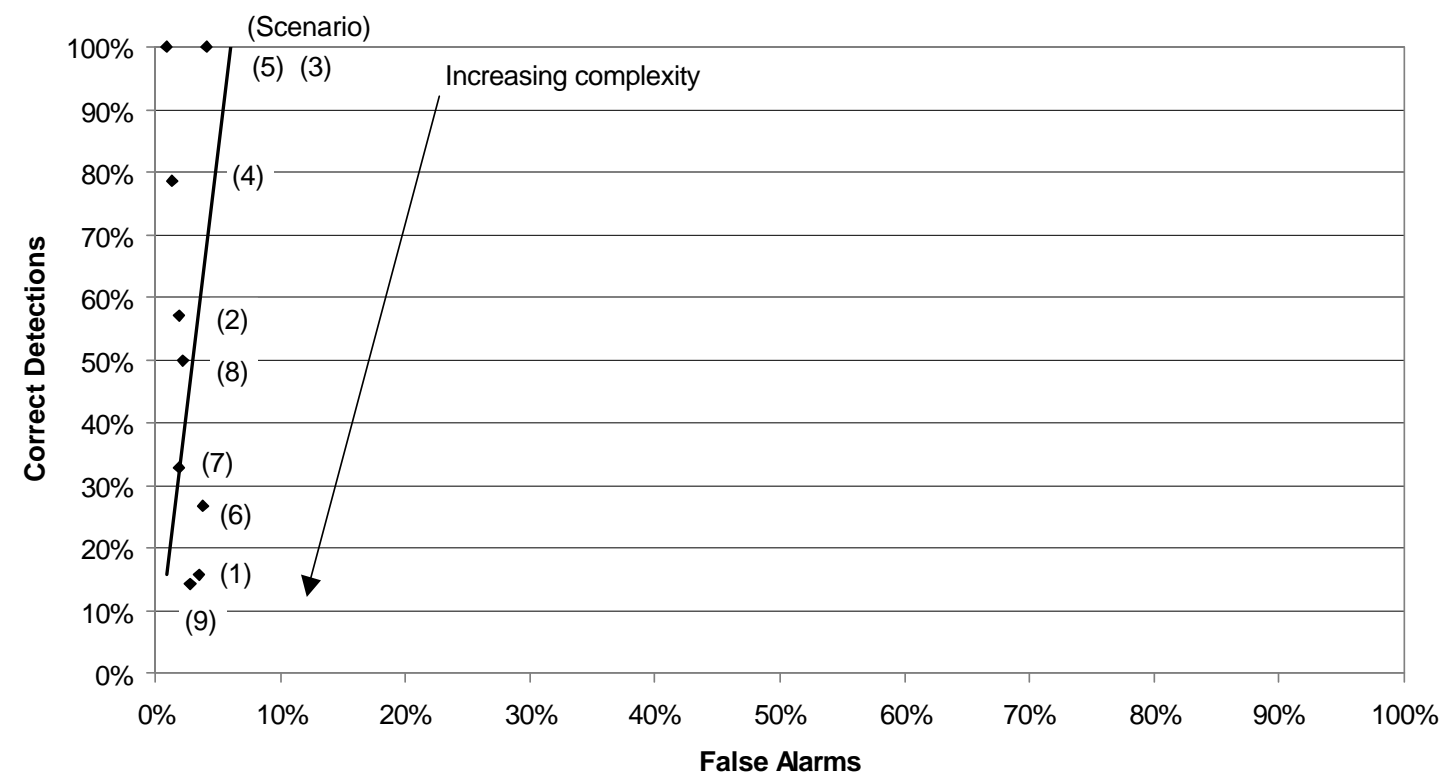

Figure 2. Receiver operating characteristic curve

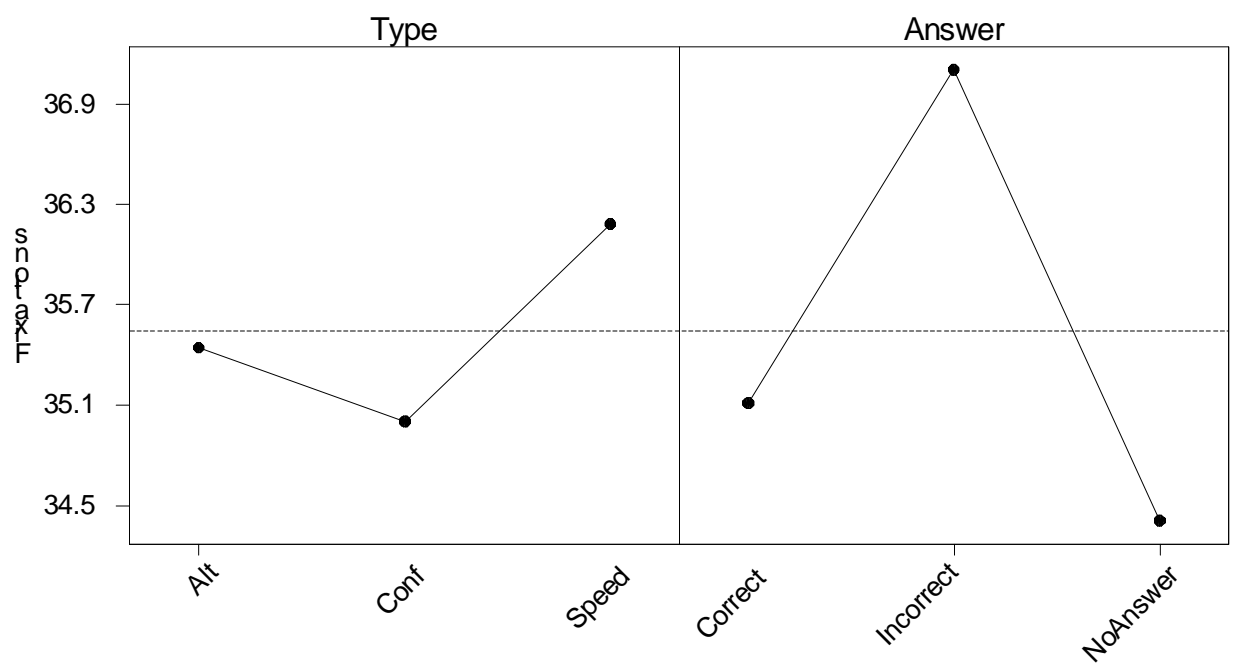

Figure 3. Main effects plot for prediction of fixations by question type and answer.

and is used here in that context [16]. Although the curve indicates high performance in general, it is where on the curve the subject is operating that is of concern. Subjects are valuing correct rejections above correct detections. This results in few false alarms at the cost of high missed detections. Most of the points should have a near perfect correct detection rate, at the expense of higher false alarms.
In a real ATC task, false alarms are much less costly than missed detections.

Recall was also very poor, in both the general and specific questions. In the specific questions, most were not answered. When they were answered, there were more right answers than wrong, most distinctly for the conflict question. Only $3 \%$ of the questions were answered wrong for the conflict question (compared to $15 \%$ for the other questions). 
So although conflicts were not recalled more often than other pieces of information, they were recalled more accurately.

General recall was very poor for questions about direction of flight, speed and altitude. The only questions answered correctly more than $50 \%$ of the time asked about the number of conflict pairs, the number of targets accepted and in what direction the targets were moving the fastest.

Interestingly, subjects correctly answered the number of conflict pairs $68 \%$ of the time, but only answered where on the screen those conflicts were $33 \%$ of the time. Air traffic controllers have been shown to have excellent spatial memory for target position. It may be that controllers obtain this ability during training, or that this is a necessary talent to be a successful air traffic controller.

Another interesting observation is that subjects knew how many targets were handed off $64 \%$ of the time, but only recalled the number of targets accepted at $21 \%$. This is probably because acceptances were a relatively simple task, requiring only that the subject see the unaccepted target icon and click on it.

Handoffs required the subjects to monitor a target until it approached the edge of the screen, highlight the target, then select a menu item, making sure that the target then disappeared. The additional time spent dealing with a target is what seemed to enable better recall.

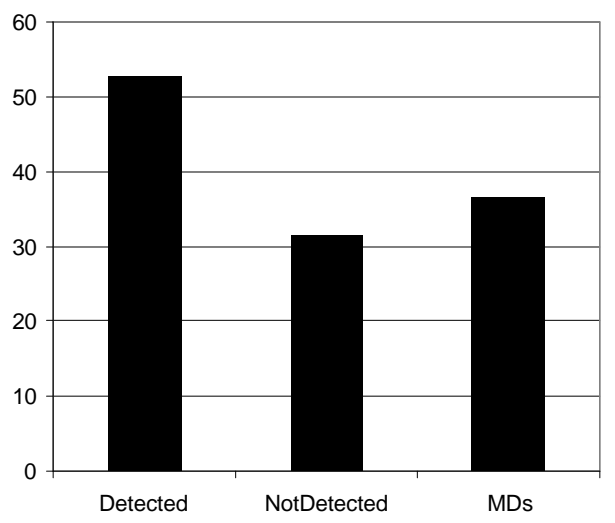

Figure 4. Fixations on conflicting targets.

One might speculate that the reason for poor recall is that subjects do not fixate on targets sufficiently. An analysis of variance (ANOVA) was performed to see if the number of fixations was dependent upon type of question (altitude question, conflict question, and speed question) and/or the answer to the question (correct answer, incorrect answer, no answer). The main effects plot from the
ANOVA is shown in Figure 3. Neither effect was statistically significant (both $p$-values were greater than 0.7). Subjects fixate as frequently on targets for which they had poor or no recall as those on which they had good recall.

The average number of fixations was greater for targets selected as conflicts as compared to those not selected as conflicts, as shown in Figure 4. However, an ANOVA performed on the data shows that this difference is not statistically different (p-value is greater than 0.25).

A more interesting statistic when examining conflict pairs is transitions. Pairs detected as conflicts had more transitions between them on average compared to pairs not detected (Figure 5), although again this difference is not statistically different (pvalue for the ANOVA was greater than 0.20) because of the high variance of the transitions.

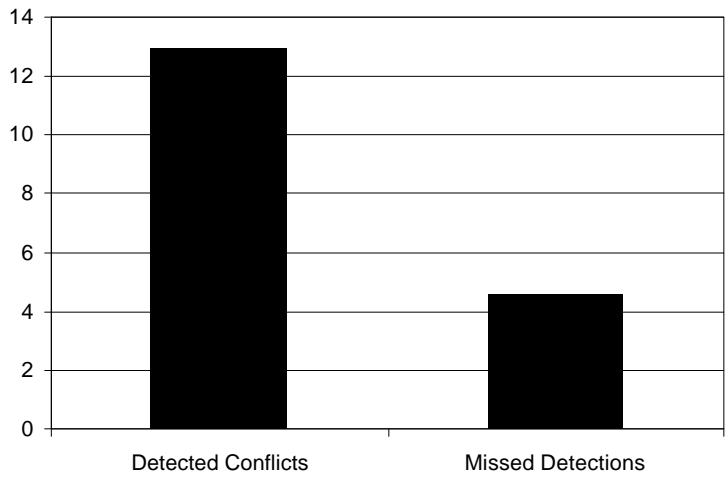

Figure 5. Transitions between conflicting pairs.

The VAN software extends the transition analysis to virtual clusters, giving a more complete picture of the pattern of fixations. The data from the eye tracker was used as input for the VAN software, which computed clusters. The clusters were then examined for the presence of Prägnanz factors (belonging to a canonical or sequenced group), proximity of targets, number of co-altitude pairs and the prevalence of detected conflict pairs within the cluster. The first two factors would indicate Gestalt groupings; the latter two would indicate goal-oriented clustering.

The number of targets that belonged to a specific sequence or canonical form within a cluster was calculated, and the average of these calculations is the "Actual" column in Figure 6. In several scenarios there was more than one sequence or canonical form, and the targets had to be of the same sequence or form to be counted. The expected percentage was calculated by taking the percentage of 
targets for the scenario that belonged to the canonical or sequenced group, then multiplying it by the size of the cluster. This provided the number of targets within each cluster one would expect to find based on random chance. The percentage was found by summing over all clusters and dividing by the total number of targets within the clusters.

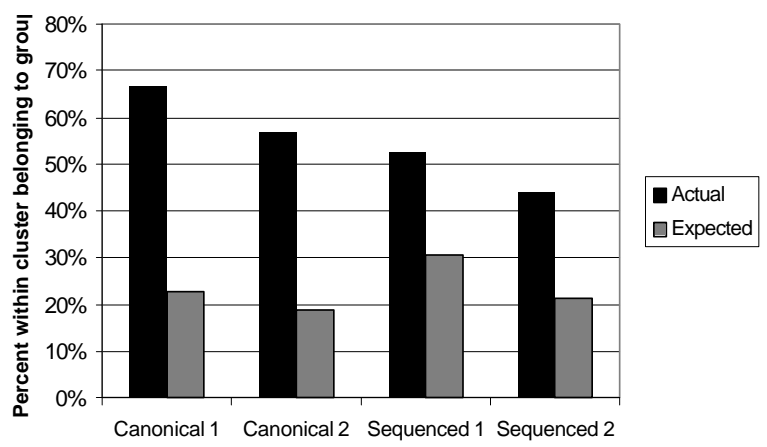

Figure 6. Average percent of cluster belonging to Prägnanz category

Chi-square tests were done on the data to see if the differences in actual versus expected were statistically significant. For the Prägnanz factors, the presence of canonical and sequenced clusters within clusters were significantly more than expected values from a random gaze hypothesis (p-value of the test was less than 0.001 in both cases).

This suggests that Prägnanz factors are a significant factor in the formation of virtual clusters. On average, Prägnanz factors are found 2.4 times more often than expected. In the canonical forms this figure is much higher.

Next the role of spatial proximity was examined. The average separation between all pairs of targets was calculated and compared with the average separation of pairs within the clusters. Pairs of targets were used since proximity is (at least) between pairs of targets. If spatial proximity were a factor in grouping, then the average separation of pairs within clusters would be smaller than the average separation of all pairs. As can be seen in figure 7, this is the case. A Chi-square test was again performed, and the proximity of targets within a cluster was significantly less than expected from a random gaze hypothesis ( $\mathrm{p}$-values of the test were less than 0.001).

A common technique discussed by controllers is to partition traffic into co-altitude groups of aircraft. This is an example of goal-oriented clustering, since the only targets that could possibly conflict are coaltitude targets. To determine if the subjects were grouping the targets in this manner, each cluster was

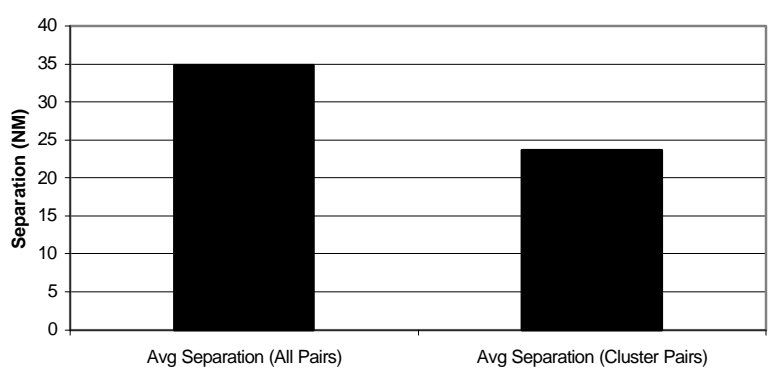

Figure 7. Average separations of pairs.

examined for the presence of co-altitude targets. Each target could be at one of five altitudes, with some altitudes more prevalent in some scenarios than others. If this type of clustering were occurring, one would expect to find a higher percentage of coaltitude targets within each cluster than one would find based on random chance. Figure 8 shows that the actual percentage of co-altitude targets within clusters is nearly identical to the expected value of co-altitude targets. A Chi-square test showed that there was no statistical difference $(\mathrm{p}=0.78)$ when compared to expected values under a random gaze hypothesis. Apparently, at least for these inexperienced ATC subjects, their gaze transitions could not ignore targets separated by significant altitude (and therefore not in danger of conflict).

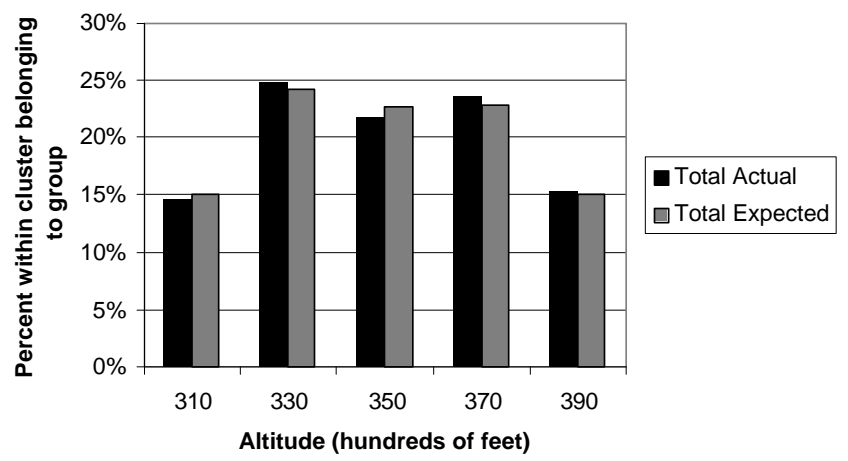

Figure 8. Percent of co-altitude targets in clusters.

Another possible goal-oriented clustering technique would be grouping by conflict pairs. Since conflicting pairs often belong to another group (proximity, sequenced, co-altitude), it is somewhat difficult to separate out this factor. In figure 9, the percentage of correctly detected conflict pairs that are found within the same cluster is shown. Also shown are incorrectly detected conflict pairs (false alarms), correctly rejected pairs, and missed detections.

Figure 9, as expected, shows a higher percentage of detected pairs within clusters than pairs not detected, particularly for correctly detected pairs. The line in 


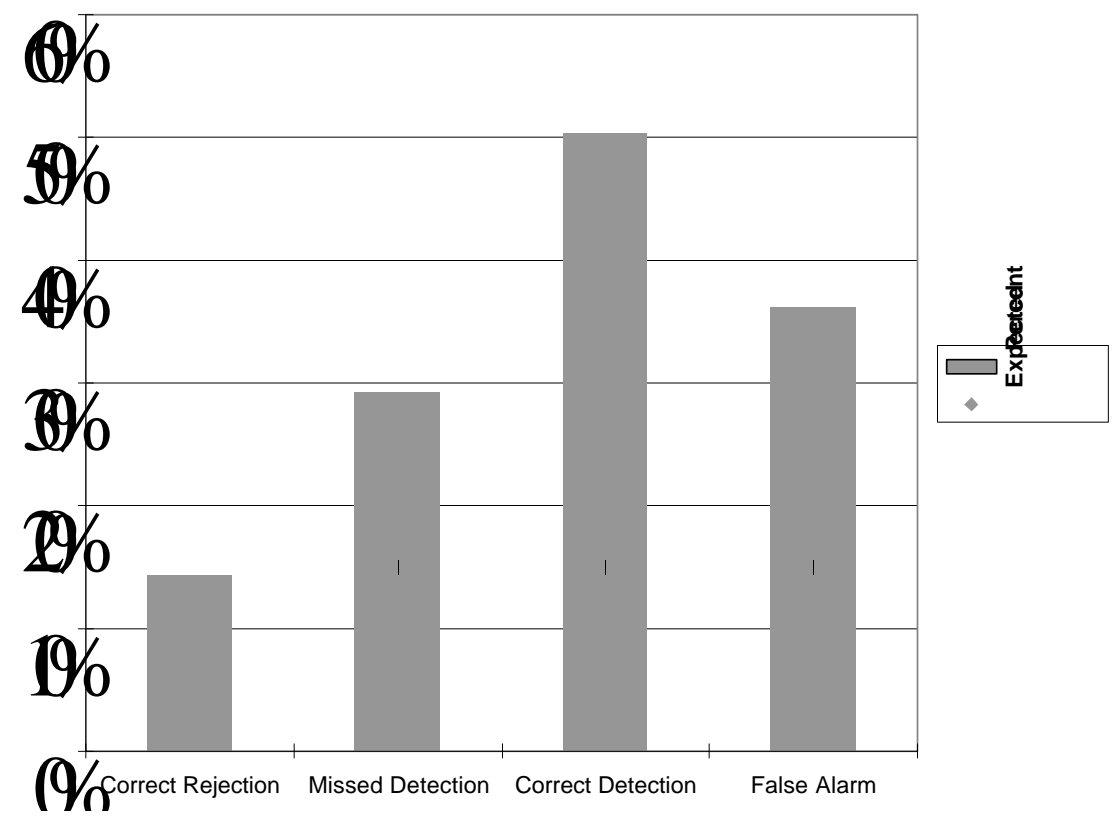

Figure 9. Percent of pairs in clusters.

figure 9 is the expected number of pairs from any particular category one would expect to randomly find for random gazes. This is the percentage of all pairs found within clusters. A Chi-square test performed showed these differences to be significant (p-value of the test $=0.001$ ).

\section{Discussion}

One hypothesis as to why air traffic control errors are made is that it is somehow due to "situational awareness" [8]. Performance on the recall task, which is often used as a measure of situational awareness, was generally poor. Specific information was only recalled about $35 \%$ of the time. Of that $35 \%$, speed and altitude information was recalled accurately $21 \%$ of the time, while conflict information was better at $30 \%$. It may seem that the poor recall and poor performance are somehow linked, and the situational awareness hypothesis is correct. However, previous practice trials with better-trained subjects, who were familiar with both the software and ATC techniques, showed better performance and equally poor recall. Also, during some informal conversations controllers were asked what information they could remember should the screen go blank, and they indicated that they could recall very little information.

Furthermore, the recall and fixation data suggests that recall is dependent upon the task and is not a primary factor in the fixation pattern. The pattern of fixations and saccades is too short to transfer information into long-term memory, as the iconic memory is constantly being erased by subsequent fixations on the same type of data. This is also supported by the finding that subjects recalled information about things that required more handling and more physical action than those that only required scanning, a result well documented by supervisory control literature [17].

The scan pattern may be able to elicit cognitive groupings, however, as discussed earlier. This idea would be strengthened by data that showed that the patterns of fixations were non-random and somehow related to the features of the task which are known to influence cognitive groupings.

The statistical data from this experiment concerning this is mixed. There was no statistical difference between transitions between conflicting and nonconflicting targets, which would be expected. However, the VAN software constructed hypothetical groupings that were nonrandom, and were influenced by Gestalt features of the task environment, which is one of the influences on cognitive grouping found by Yantis. In particular, targets related by Gestalt factors, such as common fate (sequenced), Prägnanz (canonical), and proximity were found in greater numbers within clusters as compared to random-gaze expected values. Other overlapping data, such as crossing paths (regardless of altitude), were subjectively found to be present frequently as well.

In addition to Gestalt factors, Yantis found that "goal-oriented" factors played a role in perceptual grouping. Controllers have reported using an altitudeparsing scheme when scanning for conflicts, so coaltitude pairs or groups, and pairs or groups detected as conflicts, should appear frequently if goal-oriented clustering is occurring.

This was (mostly) not the case. Co-altitude pairs were found only as often as expected from a random gaze hypothesis. Detected conflicts were found within clusters more often than expected froma random gaze hypothesis, but the extent to which this 
is due to the proximity of targets and other Gestalt factors is not entirely clear. So this result is somewhat obscured, but may be an indication of goal-based clustering if confirmed in another experiment. However, the finding that $50 \%$ of the correctly detected pairs were found within the same cluster, while only $30 \%$ of the missed detection pairs were found is important. This indicates that the ability to detect a conflict may be affected by the ability to group the conflicting targets within a cluster. Gestalt factors, having an important role in determining clusters, can interfere with the ability of the controller to form "valuable" clusters that contain conflicting pairs.

\section{CONCLUSIONS}

Our target-tracking experiment attempted to find evidence of clustering in eye movements, which previously have been shown to represent internal models of viewed scenes. The clustering revealed with the help of a novel clusterization model was dominated by Gestalt factors. Evidence of clustering based on conflict pairs was seen, although no evidence of any other goal-oriented clustering was found. Furthermore, the presence within a cluster of both targets in a conflict pair was an important factor in whether that conflict pair would be detected. If conflict pair members are also members of a Gestalt cluster, it is much more likely that the conflict will be detected. Alternately, Gestalt factors can interfere in detecting a conflict if the targets are not members of the same cluster.

The task was a slightly more complex version of previous target tracking tasks, and was similar in some ways to air traffic control. Since the subjects were not ATC professionals, the extent to which these results hold for trained controllers needs to be examined. It is expected that to some extent training can overcome Gestalt effects and the inability to cluster by altitude. Gestalt factors, however, are an innate clustering strategy and would be difficult to eliminate altogether.

These results have implications for future studies concerning virtual clustering in complex tracking tasks such as air traffic control. Air traffic is currently highly structured, and undoubtedly this structure improves the ability of the controller to detect potential conflicts. An accepted proposal for future capacity improvements is the adoption of "free flight", which could eliminate some of this structure. The extent to which controllers rely on this structure to "see" conflicts is not understood, and the lack of this structure could compromise safety. If virtual clustering is occurring, the ability to predict/detect these clusters could be invaluable.
Another conclusion drawn from our experiment is that the method of accomplishing the primary task of detecting conflicts interferes with the ability of the controller to recall details of individual targets. If the controller interacts with the target in a more physical way, by accepting the target, or handing off the target, or in some other way more significant than providing separation assurance, the target's details appear to be more susceptible to recall.

Finally we conclude that the "virtual associative network" model has proven applicable and valid for generalizing from eye-scan data for complex target tracking tasks.

\section{REFERENCES}

[1] G. A. Miller, The magical number seven, plus or minus two: some limits on our capacity for processing information. Psychological Review, vol. 63, pp. 81-97, 1953.

[2] W. A. Bousfield, The occurrence of clustering in recall of randomly arranged associates. Journal of General Psychology, vol. 49, pp. 229-240, 1953.

[3] H. Proshansky and G. Murphy, The effects of reward and punishment on perception. Journal of Experimental Psychology, vol. 13, pp. 295-305, 1942.

[4] Z. W. Pylyshyn and R. W. Storm, Tracking multiple independent targets: evidence for a parallel tracking mechanism. Spatial Vision, vol. 3, pp. 179-197, 1988.

[5] S. Yantis, Multi-element visual tracking: attention and perceptual organization. Cognitive Psychology, vol. 24, pp. 295-340, 1992.

[6] B. Means, R. J. Mumaw, C. Roth, M. S. Schlager, E. McWilliams, E. Gagne, V. Rice, D. Rosenthal and S. Heon, "ATC Training Analysis Study: Design of the Next Generation ATC Training System,” FAA/OPM 342-036, 1988.

[7] M. R. Dougherty, J. L. Perry, D. D. Ohrt, S. D. Gronlund and C. A. Manning. "Use of spatial memory by air traffic controllers," submitted for publication.

[8] M. R. Endsley and M. D. Rodgers, "Distribution of attention, situational awareness and workload in air traffic control: implications for operational errors and automation," submitted for publication.

[9] L. W. Stark and Y. S. Choi, "Experimental metaphysics: the scanpath as an epistemological mechanism," in Visual Attention and Cognition, W. H. Zangmeister, H. S. Stiehl, and C. Freksa, Eds., 1996.

[10] D. Noton and L. Stark, "Scanpaths in eye movements during pattern perception," Science, vol. 171, pp. 308-311, 1971.

[11] Eye Movements \& Scanpaths, C. Privitera. (1998). [Online]. Available: http://scan.berkeley.edu /research/eye/eye.stm.

[12] S. A. Brandt, L. Stark, S. Hacisalihzade and J. Allen, "Experimental evidence for scanpath eye movements during visual imagery," in Images of the Twenty-first Century: Proceedings of the Annual International Conference of the IEEE Engineering in Medicine and Biology Society, vol. 1, Y. Kim and F.A. Spelman, Eds., 1989, pp. 278-279.

[13] Y. M. Yufik, "Virtual Associative Network: a framework for cognitive modeling," in Brain and Values, K. H. Pribram, Ed.: Lawrence Erlbaum Associates, 1998, pp. 109 - 177.

[14] _ "A system for probabilistic resource allocation with self-adaptive capabilities,” U.S. Patent 5586219, 1996.

[15] Y. M. Yufik and T.B. Sheridan, "Virtual networks: new framework for operator modeling," Annual Reviews in Control, vol. 20, pp. 179-195, 1996. 
[16] J. Kuchar, "A Unified Methodology for the Evaluation of Hazard Alerting Systems," Ph.D. dissertation, Massachusetts Institute of Technology, Cambridge, 1995.

[17] T. B. Sheridan, Telerobotics, Automation, and Human Supervisory Control. Cambridge, MA: The MIT Press, 1992.

Steven J. Landry recently completed his S.M. from M.I.T. in the Department of Aeronautics and Astronautics. He received a B.S.E.E. from Worcester Polytechnic Institute in 1987. He was a C-141B Flight Examiner Aircraft Commander with the $18^{\text {th }}$ and $30^{\text {th }}$ squadrons at McGuire $\mathrm{AFB}, \mathrm{NJ}$ and has over 2,500 heavy jet flying hours. He is currently working on his Ph.D. at the Georgia Institute of Technology in the Department of Industrial and Systems Engineering.

Thomas B. Sheridan received a B.S.M.E. in 1951 from Purdue University, an M.S. Eng. From U.C.L.A. in 1954, and a Sc.D. in 1959 from M.I.T. He is a Professor of Engineering and Applied Psychology Emeritus in the Department of Mechanical Engineering and the Department of Aeronautics and Astronautics at M.I.T., and the head of the Human-Machine Systems Lab.

Yan M. Yufik holds Ph.D. in Physics and Applied Math, and completed post-doctoral training in cognitive and industrial psychology. He heads a research and development company, Institute of Medical Cybernetics, conducting research on cognitive modeling and decision support in complex large-scale tasks. He teaches graduate and undergraduate courses on related subjects at The Johns Hopkins University. 